\title{
A uniqueness result for scattering by infinite rough surfaces
}

Article

Published Version

Chandler-Wilde, S. N. and Zhang, B. (1998) A uniqueness result for scattering by infinite rough surfaces. SIAM Journal on Applied Mathematics (SIAP), 58 (6). pp. 1774-1790. ISSN 0036-1399 doi: https://doi.org/10.1137/S0036139996309722 Available at https://centaur.reading.ac.uk/32653/

It is advisable to refer to the publisher's version if you intend to cite from the work. See Guidance on citing.

Published version at: http://dx.doi.org/10.1137/S0036139996309722

To link to this article DOI: http://dx.doi.org/10.1137/S0036139996309722

Publisher: Society for Industrial and Applied Mathematics

All outputs in CentAUR are protected by Intellectual Property Rights law, including copyright law. Copyright and IPR is retained by the creators or other copyright holders. Terms and conditions for use of this material are defined in the End User Agreement.

\section{www.reading.ac.uk/centaur}

\section{CentAUR}

Central Archive at the University of Reading

Reading's research outputs online 


\title{
A UNIQUENESS RESULT FOR SCATTERING BY INFINITE ROUGH SURFACES*
}

\author{
SIMON N. CHANDLER-WILDE ${ }^{\dagger}$ AND BO ZHANG ${ }^{\dagger}$
}

\begin{abstract}
Consider the Dirichlet boundary value problem for the Helmholtz equation in a nonlocally perturbed half-plane with an unbounded, piecewise Lyapunov boundary. This problem models time-harmonic electromagnetic scattering in transverse magnetic polarization by one-dimensional rough, perfectly conducting surfaces. A radiation condition is introduced for the problem, which is a generalization of the usual one used in the study of diffraction by gratings when the solution is quasi-periodic, and allows a variety of incident fields including an incident plane wave to be included in the results obtained. We show in this paper that the boundary value problem for the scattered field has at most one solution. For the case when the whole boundary is Lyapunov and is a small perturbation of a flat boundary we also prove existence of solution and show a limiting absorption principle.
\end{abstract}

Key words. uniqueness, radiation condition, scattering, integral equation

AMS subject classifications. 35B40, 35L05

\section{PII. S0036139996309722}

1. Introduction. In this paper we prove a uniqueness result for the two-dimensional Dirichlet boundary value problem for the Helmholtz equation $\Delta u+k^{2} u=0$ in a nonlocally perturbed half-plane with unbounded, piecewise Lyapunov boundary. This boundary value problem arises in a study of time-harmonic acoustic scattering of an incident field by a sound-soft, infinite rough surface where the total field vanishes; the same boundary value problem (in $\mathbf{R}^{2}$ ) models two-dimensional electromagnetic scattering by a perfectly conducting, infinite, rough surface in the transverse magnetic polarization case (see [13], [18]).

The problem of scattering from a rough surface has aroused the interest of physicists, engineers, and applied mathematicians for many years, because of its large domain of application in optics, acoustics, radiowave propagation, and radar techniques. Indeed, the study of the interaction of electromagnetic waves with thin coats used in optics, the surface of the sea, or irregular terrains is the subject of numerous technical applications. Therefore, the problem has been studied by many authors using numerical or analytic methods (see, e.g., [2], [4], [16], [19] and the references quoted therein). However, very few authors have undertaken a rigorous mathematical study of the problem. Chandler-Wilde and Ross [9] established a uniqueness result for the Dirichlet problem for the Helmholtz equation in an arbitrary, unbounded domain in $\mathbf{R}^{n}(n \geq 2)$ in the case when $\Im k>0$. In [10] the same authors proved existence of a solution for the boundary value problem in $\mathbf{R}^{2}$ in the case when the whole boundary is both Lyapunov and a small perturbation of a flat boundary. They further showed, for the case $k>0$, that the solution constructed is the unique solution satisfying a limiting absorption principle. In both [9] and [10] the function space specified for the Dirichlet data is sufficiently large so that the case of plane wave incidence is included in the results obtained.

\footnotetext{
${ }^{*}$ Received by the editors September 25, 1996; accepted for publication (in revised form) February 17, 1997; published electronically August 3, 1998. This work was supported by UK Engineering and Physical Sciences Research Council grant GR/K24406.

http://www.siam.org/journals/siap/58-6/30972.html

${ }^{\dagger}$ Department of Mathematics and Statistics, Brunel University, Uxbridge UB8 3PH, UK (simon.chandler-wilde@brunel.ac.uk, bo.zhang@brunel.ac.uk).
} 
In this paper we consider the Dirichlet boundary value problem with arbitrary bounded continuous data, so that, when $k>0$, as in [9], [10], the scattering problem with plane wave incidence is within the scope of the theory developed. Our assumption that the boundary is piecewise Lyapunov includes most one-dimensional rough surfaces of practical interest. The prime concern of the paper is to propose a radiation condition for the problem and, using this radiation condition, to prove that the boundary value problem has at most one solution. Additionally, for the case when the whole boundary is Lyapunov and is a small perturbation of a flat boundary, we prove, by applying the results in [10], that a solution satisfying the radiation condition exists and, moreover, that the solution selected by the radiation condition satisfies a limiting absorption principle.

The radiation condition proposed is a generalization of the radiation condition used in the study of plane wave diffraction by one-dimensional periodic gratings [1], [17], [18]. Indeed (see [6]), the radiation condition we impose reduces to the usual one (based on an assumption of validity of a Rayleigh expansion above the grating), and thus our boundary value problem reduces to the usual formulation, if our rough surface is periodic and the solution is assumed quasi-periodic. Thus our uniqueness proof contains the uniqueness result for the periodic grating case [17] as a special case, but note that our uniqueness result for this case is obtained without any a priori assumption of quasi-periodicity.

The uniqueness proof for the corresponding diffraction grating case [17] (and see [3]) is one starting point for the proof in this paper; the proof also has ideas in common with recent uniqueness proofs for the impedance boundary value problem for the Helmholtz equation in a half-plane [7] and for the problem of electromagnetic scattering by an inhomogeneous conducting or dielectric layer on a perfectly conducting plate [11].

The outline of this paper is as follows. In the next section the scattering problem is introduced and the radiation condition discussed. Section 3 contains the uniqueness result, and in section 4, the results of [10] are applied to obtain existence of solution in certain cases. In the appendix we prove a lemma which enables, under certain conditions, square integrability on the real line to be deduced from local square integrability. This lemma plays a key role in proving the uniqueness result in section 3 and has been used similarly recently in the uniqueness proof in [11].

We conclude this section by introducing some notations. For $h \in \mathbf{R}$, define $\Gamma_{h}=\left\{x=\left(x_{1}, x_{2}\right) \in \mathbf{R}^{2} \mid x_{2}=h\right\}$ and $U_{h}=\left\{x \in \mathbf{R}^{2} \mid x_{2}>h\right\}$. Given an open set $V \subset \mathbf{R}^{2}$ and $v \in L_{\infty}(V)$, denote by $\partial_{j} v, j=1,2$, the (distributional) derivative $\partial v(x) / \partial x_{j}$. For $V \subset \mathbf{R}^{2}$ denote by $B C(V)$ the Banach space of functions bounded and continuous on $V$. Finally, for $A>0, x \in \mathbf{R}^{2}$, let $B_{A}(x)=\left\{y \in \mathbf{R}^{2}|| y-x \mid<A\right\}$.

2. The scattering problem and radiation condition. Given $f \in C(\mathbf{R})$ which satisfies, for some constants $f_{+}>f_{-}>0$,

$$
f_{-} \leq f\left(x_{1}\right) \leq f_{+}, \quad x_{1} \in \mathbf{R},
$$

define the two-dimensional region $D$ by

$$
D=\left\{x=\left(x_{1}, x_{2}\right) \in \mathbf{R}^{2} \mid x_{2}>f\left(x_{1}\right)\right\}
$$

so that the boundary $\Gamma$ of $D$ is

$$
\Gamma \equiv \partial D=\left\{\left(x_{1}, f\left(x_{1}\right)\right) \mid x_{1} \in \mathbf{R}\right\} .
$$


We consider the scattering problem of a field $u^{\mathrm{i}}$, a solution of the Helmholtz equation $\Delta u^{\mathrm{i}}+k^{2} u^{\mathrm{i}}=0$ in $D$, incident on the infinite boundary $\Gamma$. We assume that $k$ is a complex constant with $\Im k \geq 0, \Re k>0$ and restrict our attention to the case when the total field vanishes on the boundary, so that the scattered field $u$, also a solution of the Helmholtz equation in $D$, satisfies the Dirichlet boundary condition $u=-u^{\mathrm{i}}$ on $\Gamma$.

Throughout we assume that $f$ is Lipschitz continuous, i.e., that, for some positive constant $L>0$,

$$
|f(s)-f(t)| \leq L|s-t|, \quad s, t \in \mathbf{R} .
$$

This assumption implies that $f$ has a derivative in a weak sense and that $f^{\prime} \in L_{\infty}(\mathbf{R})$ with $\left\|f^{\prime}\right\|_{\infty} \leq L$. We assume further that $f^{\prime}$ is piecewise continuous, precisely that there exists a set $T=\left\{t_{j} \mid j \in \mathbf{Z}\right\}$, with $\cdots<t_{-1}<t_{0}<t_{1}<\cdots$ and with $T$ having no finite limit points, such that $f^{\prime}$ is Hölder continuous on $\left[t_{j-1}, t_{j}\right]$ for every $j \in \mathbf{Z}$. This assumption implies that $\Gamma$ is piecewise Lyapunov and that the unit normal vector $n(x)=\left(n_{1}(x), n_{2}(x)\right)$, directed out of $D$, exists at every point $x \in \Gamma \backslash S$, where $S=\left\{\left(x_{1}, f\left(x_{1}\right)\right) \mid x_{1} \in T\right\}$ is the set of corners of $\Gamma$. From (2.1) we see that the corner angle exterior to $D$ at each point $x \in S$ is $\geq \theta$, where

$$
\theta=2 \tan ^{-1}(1 / L) .
$$

In order for the problem to have a unique solution, a radiation condition as $x_{2}$ tends to infinity has to be imposed on the scattered field $u$; that is, the scattered field $u$ should behave as an outgoing wave as $x_{2} \rightarrow+\infty$. We wish to include in our consideration incident fields including the incident plane wave so that the standard Sommerfeld radiation condition is not appropriate in this context as we cannot expect that $u$ will decay in the $x_{1}$ direction. We will use the radiation condition proposed in [7] and utilized recently in [11], which we will refer to as the upward propagating radiation condition, and will usefully relate this condition to the Sommerfeld radiation condition. To this end introduce the following definitions.

Definition 2.1. Given a domain $G \subset \mathbf{R}^{2}$, call $v \in C^{2}(G) \cap L_{\infty}(G)$ a radiating solution of the Helmholtz equation in $G$ if $\Delta v+k^{2} v=0$ in $G$ and

$$
\begin{array}{r}
v(x)=O\left(r^{-1 / 2}\right), \\
\frac{\partial v(x)}{\partial r}-i k v(x)=o\left(r^{-1 / 2}\right)
\end{array}
$$

as $r=|x| \rightarrow \infty$, uniformly in $x /|x|$.

Let $\Phi(x, y)$ denote the free-space Green's function for $\Delta+k^{2}$, that is,

$$
\Phi(x, y)=\frac{i}{4} H_{0}^{(1)}(k|x-y|), \quad x, y \in \mathbf{R}^{2}, \quad x \neq y,
$$

where $H_{n}^{(1)}$ is the Hankel function of the first kind of order $n$.

Definition 2.2. Given a domain $G \subset \mathbf{R}^{2}$, say that $v: G \rightarrow \mathbf{C}$ satisfies the upward propagating radiation condition (UPRC) in $G$ if, for some $h \in \mathbf{R}$ and $\phi \in$ $L_{\infty}\left(\Gamma_{h}\right), U_{h} \subset G$ and

$$
v(x)=2 \int_{\Gamma_{h}} \frac{\partial \Phi(x, y)}{\partial y_{2}} \phi(y) d s(y), \quad x \in U_{h} .
$$


Note that the existence of the integral in (2.3) for arbitrary $\phi \in L_{\infty}\left(\Gamma_{h}\right)$ is assured by the bound which follows from the asymptotic behavior of the Hankel function for small and large argument,

$$
\left|\frac{\partial \Phi(x, y)}{\partial y_{2}}\right| \leq C\left|x_{2}-y_{2}\right|\left(|x-y|^{-2}+|x-y|^{-3 / 2}\right), \quad x, y \in \mathbf{R}^{2}, x \neq y,
$$

which holds for some constant $C>0$ dependent only on $k$.

The following result states properties of the upward propagating radiation condition needed later and shows that any radiating solution satisfies the UPRC.

Lemma 2.1 (see [11, Theorem 2.1]). Given $H \in \mathbf{R}$ and $v: U_{H} \rightarrow \mathbf{C}$, the following statements are equivalent:

(i) $v \in C^{2}\left(U_{H}\right), v \in L_{\infty}\left(U_{H} \backslash U_{a}\right)$ for all $a>H, \Delta v+k^{2} v=0$ in $U_{H}$, and $v$ satisfies the UPRC in $U_{H}$;

(ii) there exists a sequence $\left(v_{n}\right)$ of radiating solutions such that $v_{n}(x) \rightarrow v(x)$ uniformly on compact subsets of $U_{H}$ and

$$
\sup _{x \in U_{H} \backslash U_{a}, n \in \mathbf{N}}\left|v_{n}(x)\right|<\infty
$$

for all $a>H$

(iii) $v$ satisfies (2.3) for $h=H$ and some $\phi \in L_{\infty}\left(\Gamma_{H}\right)$;

(iv) $v \in L_{\infty}\left(U_{H} \backslash U_{a}\right)$ for some $a>H$ and $v$ satisfies (2.3) for each $h>H$ with $\phi=\left.v\right|_{\Gamma_{h}} ;$

(v) $v \in C^{2}\left(U_{H}\right), v \in L_{\infty}\left(U_{H} \backslash U_{a}\right)$ for all $a>H, \Delta v+k^{2} v=0$ in $U_{H}$, and, for every $h>H$ and radiating solution in $U_{H}, w$, such that the restrictions of $w$ and $\partial_{2} w$ to $\Gamma_{h}$ are in $L_{1}\left(\Gamma_{h}\right)$, it holds that

$$
\int_{\Gamma_{h}}\left(v \frac{\partial w}{\partial n}-w \frac{\partial v}{\partial n}\right) d s=0
$$

In that the above lemma shows that any radiating solution satisfies the UPRC, this radiation condition is less restrictive than the Sommerfeld radiation condition. Further, the UPRC generalizes the standard radiation condition for one-dimensional periodic gratings. Precisely, it is shown in [6] that if $v$ has the usual representation as a Rayleigh expansion [1], [17], [18] in some half-plane $U_{H}$, then it also satisfies (2.3) for all $h>H$ and so satisfies the UPRC. As a consequence, any upward propagating homogeneous or inhomogeneous plane wave satisfies (2.3).

To further motivate the proposed radiation condition we point out that it can be viewed as a rigorous formulation of a radiation condition often imposed in a nonrigorous manner in treatments of rough surface scattering. It is common to require (e.g., [12]) that, in some upper half-plane $U_{h}$ with $h>f_{+}$, the scattered field $v$ has a representation as a superposition of upward propagating homogeneous and inhomogeneous plane waves, precisely that

$$
v(x)=\frac{1}{2 \pi} \int_{-\infty}^{+\infty} \exp \left(i\left[\left(x_{2}-h\right) \sqrt{k^{2}-\xi^{2}}+x_{1} \xi\right]\right) \hat{F}(\xi) d \xi, \quad x \in U_{h},
$$

where $\sqrt{k^{2}-\xi^{2}}=i \sqrt{\xi^{2}-k^{2}},|\xi|>k$, and $\hat{F}$ is the Fourier transform of $F=\left.u\right|_{\Gamma_{h}}$. If $F \in L_{2}(\mathbf{R})$ (in which case $\hat{F} \in L_{2}(\mathbf{R})$ ) it follows from standard results on Fourier transforms of convolutions [7] that (2.3) and (2.7) are equivalent; the right-hand sides 
of (2.3) and (2.7) are identical if we set $\phi=F$ in (2.3). But it is common, as in [12], to impose (2.7) also in cases when an incident plane wave is under consideration when it is reasonable to suppose that $F \in L_{\infty}(\mathbf{R})$ rather than $F \in L_{2}(\mathbf{R})$. In this case $\hat{F}$ exists in general only as a tempered distribution so that the integral in (2.7) does not have a meaning in a classical sense. Moreover, if $k>0,(2.7)$ is not defined either in the generalized sense of the Fourier transform of a tempered distribution (the difficulty being that the product of $\exp \left(i\left(x_{2}-h\right) \sqrt{k^{2}-.^{2}}\right)$ with a tempered distribution does not have a standard definition unless $\Im k>0$ ). Equation (2.3) can be viewed as giving a meaning to (2.7) in this case.

The scattering problem of an incident field by an infinite rough surface can now be formulated as the following boundary value problem for the scattered field $u$. The function space specified for the Dirichlet data $g$ includes, when $k>0$, the usual incident fields of interest including the incident plane wave.

Problem (P). Given $g \in B C(\Gamma)$, determine $u \in C^{2}(D) \cap C(\bar{D})$ such that

(i) $u$ is a solution of the Helmholtz equation, i.e.,

$$
\Delta u+k^{2} u=0 \text { in } D ;
$$

(ii) $u=g$ on $\partial D$;

(iii) for some $\beta \in \mathbf{R}$,

$$
\sup _{x \in D} x_{2}^{\beta}|u(x)|<\infty
$$

(iv) $u$ satisfies the upward propagating radiation condition (2.3).

It follows from [7, Theorem 3.1] that, if $\Im k>0$, the radiation condition (iv) is superfluous in that it follows from the other conditions assumed; and the uniqueness result in [9] establishes that Problem $(\mathrm{P})$ has at most one solution in this case. The next section is concerned with establishing this uniqueness result for the case $k>0$. Conditions (i)-(iii) alone do not then guarantee uniqueness as is shown by the simple example $u(x)=\sin \left(k\left(x_{2}-1\right)\right)$, which satisfies Problem (P) in the case of the flat boundary $f \equiv 1$, with $g \equiv 0$ and $\beta=0$ in (2.9).

3. Uniqueness for the case $\boldsymbol{k}>\mathbf{0}$. Suppose that $u_{1}$ and $u_{2}$ are solutions of Problem (P). Then $u=u_{1}-u_{2}$ satisfies Problem (P) with $g=0$. Therefore, in order to prove that Problem $(\mathrm{P})$ has at most one solution, it is enough to show that Problem (P) with $g \equiv 0$ has only the trivial solution. Throughout this section, set $g \equiv 0, k>0$, and we are concerned with showing that Problem (P) then has only the trivial solution. Note that in this section we will abbreviate $\partial u / \partial n$, the normal derivative of $u$, as $\partial_{n} u$.

3.1. A priori estimates. Suppose that $u \in C^{2}(D) \cap C(\bar{D})$ satisfies conditions (i)-(iii) of Problem (P) (with $g \equiv 0, k>0$ ). Then, by standard elliptic regularity estimates [14, section 8.11], $u \in C^{1}(\bar{D} \backslash S)$ with $S$ being the set of corners of $\Gamma$, since $\Gamma$ is piecewise Lyapunov. In order to prove the uniqueness result it is necessary to establish a priori estimates for $u$ and its gradient up to the boundary. Our argument uses ideas in [20], where bounds on the Dirichlet Green's function for the operator $\Delta$ in bounded regions with Lyapunov boundary are obtained.

Before going further we introduce some notations which are used in the remaining part of the paper. For $A, B \in \mathbf{R}$ with $A>B$ and subset $V \subset \mathbf{R}^{2}$, define $V(B, A)=$ $\left\{x \in V \mid B<x_{1}<A\right\}$, and write $\Gamma_{h}(A)=\Gamma_{h}(-A, A), \Gamma(A)=\Gamma(-A, A)$. For $h>f_{+}$, define $D_{h}=D \backslash \bar{U}_{h}$, and write $D_{h}(A)=D_{h}(-A, A)$. 
In the proof of Theorem 3.1 below we use the following interior regularity estimate [14, Theorem 3.9].

Lemma 3.1. If $G \subset \mathbf{R}^{2}$ is open and bounded, $v \in C^{2}(G) \cap C(\bar{G}), f \in C(\bar{G})$, and $\Delta v=f$ in $G$, then

$$
|\nabla v(x)| \leq \tilde{C}(d(x))^{-1}\left(\|v\|_{\infty}+\left\|d^{2} f\right\|_{\infty}\right), \quad x \in G,
$$

where $\tilde{C}$ is an absolute constant and $d(x)=\operatorname{dist}(x, \partial G)$.

Applying this lemma with $G=B_{\eta}(x)$ and $\eta$ some sufficiently small positive constant dependent on the value of $\epsilon$ in the bound (3.1) below, we see immediately that $\nabla u$ satisfies the same bound (2.9) as $u$ in the interior of $D$, precisely

$$
\sup _{x_{1} \in \mathbf{R}, x_{2}>f\left(x_{1}\right)+\epsilon} x_{2}^{\beta}|\nabla u(x)|<\infty
$$

for all $\epsilon>0$. The following result gives sharper bounds on $u$ and $\nabla u$ in a neighborhood of the boundary of $D$. The angle $\theta$ is as defined in (2.2).

Theorem 3.1. If $u \in C^{2}(D) \cap C(\bar{D})$ satisfies conditions (i)-(iii) of Problem (P) (with $g \equiv 0, k>0$ ), then, for some positive constant $C$,

$$
\begin{aligned}
|u(x)| & \leq C\left[x_{2}-f\left(x_{1}\right)\right]^{\alpha}, \\
|\nabla u(x)| & \leq C\left[x_{2}-f\left(x_{1}\right)\right]^{\alpha-1}
\end{aligned}
$$

for $x \in D_{B}$, where $B=f_{+}+1$ and $1 / 2<\alpha=\pi /(2 \pi-\theta)<1$.

Proof. Let $E=2+f_{+}-f_{-}$, and let us define $G$ by

$$
G:=\left\{\left(x_{1}, x_{2}\right)|| x_{1}|<E / L,-L| x_{1} \mid<x_{2}<E\right\} .
$$

Define $w \in C^{2}(G) \cap C(\bar{G})$ as satisfying $\Delta w=k^{2}$ in $G$ and $w=h$ on $\partial G$, where $h \in C(\partial G)$ is chosen so that $-1 \leq h \leq 0, h=-1$ on $\partial G_{1}=\left\{x|| x_{1} \mid=E / L, 1-E \leq\right.$ $\left.x_{2} \leq E\right\} \cup\left\{x|| x_{1} \mid \leq E / L, x_{2}=E\right\}, h=0$ on $\partial G_{2}=\left\{x|| x_{1}\left|\leq E / L, x_{2}=-L\right| x_{1} \mid\right\}$. Then, by the elliptic singularity theory (see, e.g., [15]), we obtain that, for some $K>0$,

$$
|w(x)| \leq K|x|^{\alpha}, \quad x \in \bar{G} .
$$

Further, by the maximum principle, $w \leq 0$ in $\bar{G}$.

Let $b=f_{+}+E$ and $C=\sup _{x \in D_{b}}|u(x)|$. For $x \in \Gamma$ let $G_{x}=G+x=\{y+x \mid y \in G\}$, and define $w_{x} \in C\left(\bar{G}_{x}\right) \cap C^{2}\left(G_{x}\right)$ by $w_{x}(y)=C w(y-x), y \in \bar{G}_{x}$. Let $v$ denote either the real or the imaginary part of $u$. Then, defining $V=D \cap G_{x}, v \in C(\bar{V}) \cap C^{2}(V)$, $|\Delta v| \leq C k^{2},|v| \leq C$ in $V$, and $v=0$ on $\Gamma \cap \partial V$. Also, $\Delta w_{x}=C k^{2}$ in $V, w_{x}=-C$ on $\partial V \cap D$, and $w_{x} \leq 0$ on $\Gamma \cap \partial V$. Let $v_{ \pm}= \pm v+w_{x}$. Then $v_{ \pm} \leq 0$ on $\partial V, \Delta v_{ \pm} \geq 0$ in $V$, and so, by the maximum principle, $v_{ \pm} \leq 0$ in $\bar{V}$. Therefore $|v| \leq-w_{x}$ in $\bar{V}$ and thus, for $0 \leq h \leq E$,

$$
\left|v\left(x+h e_{2}\right)\right| \leq-w_{x}\left(x+h e_{2}\right)=-C w(0, h) \leq C K h^{\alpha}
$$

by (3.4). Since this holds for all $x \in \Gamma$, it follows that, for some constant $C_{1}>0$,

$$
|u(x)| \leq C_{1}\left[x_{2}-f\left(x_{1}\right)\right]^{\alpha}, \quad x \in D_{B+1} .
$$

For $x \in D_{B}$, by Lemma 3.1 ,

$$
|\nabla u(x)| \leq \tilde{C}[\eta(x)]^{-1}\left(1+k^{2}\right) \sup _{y \in B_{\eta(x)}(x)}|u(y)|,
$$


where $\eta(x)=\min (1, d(x) / 2)$ and $d(x)=\operatorname{dist}(x, \Gamma)$. Thus, in view of (3.5) and since

$$
\left(1+L^{2}\right)^{-1 / 2} \leq d(x) /\left(x_{2}-f\left(x_{1}\right)\right) \leq 1, \quad x \in D,
$$

it follows that

$$
|\nabla u(x)| \leq \tilde{C} C_{1}[\eta(x)]^{-1}\left(1+k^{2}\right)\left[3\left(x_{2}-f\left(x_{1}\right)\right) / 2\right]^{\alpha} \leq C\left(x_{2}-f\left(x_{1}\right)\right)^{\alpha-1},
$$

where $C=\tilde{C} C_{1}\left(1+k^{2}\right)(3 / 2)^{\alpha} \max \left(E-1,2\left(1+L^{2}\right)^{1 / 2}\right)$. The proof is complete.

3.2. A basic inequality. As in section 3.1 , suppose that $u \in C^{2}(D) \cap C(\bar{D})$ satisfies conditions (i)-(iii) of Problem (P) (with $g \equiv 0, k>0$ ). We prove in this section a basic inequality satisfied by $u$ which plays an important part in the proof of the uniqueness result.

Given $a>f_{+}$define, for $A, B \in \mathbf{R}$ with $A>B$,

$$
I(B, A)=\int_{\Gamma_{a}(B, A)}\left\{\left|\partial_{2} u\right|^{2}-\left|\partial_{1} u\right|^{2}+k^{2}|u|^{2}\right\} d s .
$$

For $a>f_{+}, b \in \mathbf{R}$, let $\gamma_{a}(b)=\left\{\left(b, x_{2}\right) \mid f(b) \leq x_{2} \leq a\right\}$.

TheOrem 3.2. Let $u \in C^{2}(D) \cap C(\bar{D})$ satisfy conditions (i)-(iii) of Problem (P) (with $g \equiv 0, k>0$ ). Then $\partial_{n} u \in L_{2}^{\text {loc }}(\Gamma)$ and, for some positive constant $C$,

$$
\int_{\Gamma(B, A)}\left|\partial_{n} u\right|^{2} d s \leq C\left\{I(B, A)+R_{1}(B, A)\right\}
$$

for all $A, B \in \mathbf{R}$ with $A>B$, where

$$
R_{1}(B, A)=2 \Re\left[\int_{\gamma_{a}(A)}-\int_{\gamma_{a}(B)}\right] \partial_{2} \bar{u} \partial_{1} u d s .
$$

Proof. Let $B_{j}$ denote the square with horizontal and vertical sides of length $d>0$ centered on the corner $\left(t_{j}, f\left(t_{j}\right)\right)$, i.e., $B_{j}=\left\{x \in \mathbf{R}^{2}|| x_{1}-t_{j}|<d / 2,| x_{2}-f\left(t_{j}\right) \mid<\right.$ $d / 2\}, j \in \mathbf{Z}$. Then $u \in C^{1}\left(\bar{D} \backslash S_{d}\right) \cap C^{2}(D)$, where $S_{d}=\cup_{j \in \mathbf{Z}} B_{j}$. Given $A>B$ and $a>f_{+}$, choose $d<a-f_{+}$, and set $T=D_{a}(B, A) \backslash S_{d}$. Multiply (2.8) by $2 \partial_{2} \bar{u}$, integrate over $T$, and take the real part of the equation thus obtained. Noting that $2 \Re\left\{\partial_{2} \bar{u}\left(\Delta u+k^{2} u\right)\right\}=2 \Re\left\{\nabla \cdot\left(\partial_{2} \bar{u} \nabla u\right)\right\}-\partial_{2}\left(|\nabla u|^{2}\right)+k^{2} \partial_{2}\left(|u|^{2}\right)$, we find, on applying the divergence theorem, that

$$
I(B, A)+I_{1}=I_{2}+I_{3},
$$

where

$$
\begin{aligned}
& I_{1}=2 \Re\left[\int_{\gamma_{a}(A) \backslash S_{d}}-\int_{\gamma_{a}(B) \backslash S_{d}}\right] \partial_{2} \bar{u} \partial_{1} u d s, \\
& I_{2}=\int_{\Gamma(B, A) \backslash S_{d}}\left\{n_{2}|\nabla u|^{2}-2 \Re\left(\partial_{2} \bar{u} \partial_{n} u\right)\right\} d s,
\end{aligned}
$$

and

$$
I_{3}=\int_{\partial S_{d} \cap D_{a}(B, A)}\left\{n_{2}\left(|\nabla u|^{2}-k^{2}|u|^{2}\right)-2 \Re\left(\partial_{2} \bar{u} \partial_{n} u\right)\right\} d s .
$$


As $u=0$ on $\Gamma$, we have that $\partial_{2} u=n_{2} \partial_{n} u$ and $|\nabla u|=\left|\partial_{n} u\right|$ on $\Gamma \backslash S$. Since also $n_{2}=-\left\{1+\left[f^{\prime}\right]^{2}\right\}^{-1 / 2} \leq-\left(1+L^{2}\right)^{-1 / 2}$ on $\Gamma \backslash S$, it follows that

$$
I_{2}=-\int_{\Gamma(B, A) \backslash S_{d}} n_{2}\left|\partial_{n} u\right|^{2} d s \geq\left(1+L^{2}\right)^{-1 / 2} \int_{\Gamma(B, A) \backslash S_{d}}\left|\partial_{n} u\right|^{2} d s .
$$

Now, using Theorem 3.1, we easily see that $R_{1}(B, A)$ is well defined as an improper integral, so that $I_{1} \rightarrow R_{1}(B, A)$ as $d \rightarrow 0$. Further, from Theorem 3.1, we easily estimate that, for some constant $C>0,\left|I_{3}\right| \leq C d^{2 \alpha-1}$. Thus $I_{3} \rightarrow 0$ as $d \rightarrow 0$, since $\alpha>1 / 2$. It follows, on letting $d \rightarrow 0$ in (3.11) and noting (3.12), that $\partial_{n} u \in$ $L_{2}(\Gamma(B, A))$ and that (3.10) holds. Since $B$ and $A$ are arbitrary, $\partial_{n} u \in L_{2}^{l o c}(\Gamma)$.

Taking $A=j$ and $B=j-1$ with $j \in \mathbf{Z}$ in (3.10) we have, on using (2.9), (3.1), and Theorem 3.1 to bound $R_{1}(B, A)$ and $I(B, A)$ independently of $B$ and $A$, the following result.

COROLlary 3.1. Let u satisfy the conditions of Theorem 3.2. Then

$$
\sup _{j \in \mathbf{Z}} \int_{\Gamma(j-1, j)}\left|\partial_{n} u\right|^{2} d s<+\infty .
$$

3.3. A representation theorem. In this section we derive a form of Green's representation theorem for the solution $u$ of Problem $(\mathrm{P})$ in the case $g \equiv 0$, using Green's theorem combined with the radiation condition (2.3).

Let $G(x, y)$ be the Dirichlet Green's function for the Helmholtz operator $\Delta+k^{2}$ in the upper half-plane $U_{0}$. Then

$$
G(x, y)=\Phi(x, y)-\Phi\left(x, y^{\prime}\right), \quad x, y \in \overline{U_{0}}, \quad x \neq y,
$$

where $y=\left(y_{1}, y_{2}\right), y^{\prime}=\left(y_{1},-y_{2}\right)$.

From the definition of $G$ and the asymptotic properties of the Hankel function it follows that, for some constant $C>0$ independent of $x$ and $y$,

$$
|G(x, y)| \leq C(1+|\log | x-y||)
$$

and that (see [10])

$$
\begin{aligned}
|G(x, y)| & \leq \frac{C x_{2} y_{2}}{|x-y|}\left|H_{1}^{(1)}(k|x-y|)\right| \\
& \leq C x_{2} y_{2}\left\{|x-y|^{-2}+|x-y|^{-3 / 2}\right\} .
\end{aligned}
$$

Both inequalities hold for all $x, y \in \overline{U_{0}}, x \neq y$. From (3.16) and Lemma 3.1 it follows that, given $C>0$,

$$
G(x, y), \nabla_{y} G(x, y)=O\left(|x-y|^{-3 / 2}\right)
$$

uniformly in $x$ and $y$ with $0 \leq x_{2}, y_{2} \leq C$ as $|x-y| \rightarrow \infty$, and that, given $a>f_{+}$,

$$
|G(x, y)|, \quad\left|\nabla_{x} G(x, y)\right|, \quad\left|\nabla_{y} G(x, y)\right| \leq C_{a}\left(1+\left|x_{1}-y_{1}\right|\right)^{-3 / 2}
$$

for $y \in \Gamma, x \in \Gamma_{a}$, where $C_{a}$ is a positive constant depending only on $a$ and $k$.

TheOREM 3.3. Let $u$ be a solution of Problem $(\mathrm{P})$ (with $g \equiv 0$ ). Then

$$
u(x)=\int_{\Gamma} \partial_{n} u(y) G(x, y) d s(y), \quad x \in D .
$$


Proof. Define $S_{d}$ as in the proof of Theorem 3.2. Take $x \in D$, and choose $a>\max \left(x_{2}, f_{+}\right), A>\left|x_{1}\right|, d$ in the range $0<d<x_{2}-f\left(x_{1}\right)$, and $\epsilon>0$ sufficiently small. Noting that (see section 3.1) $u \in C^{1}(\bar{D} \backslash S)$, apply Green's second theorem to $G(x, \cdot)$ and $u$ in the bounded region $D_{a}(A) \backslash \overline{B_{\epsilon}(x) \cup S_{d}}$, and then let $\epsilon \rightarrow 0$ to obtain that

$$
u(x)=\int_{\partial T}\left[u(y) \frac{\partial G(x, y)}{\partial n(y)}-G(x, y) \frac{\partial u}{\partial n}(y)\right] d s(y)
$$

where $T=D_{a}(A) \backslash \overline{S_{d}}$. Letting first $d \rightarrow 0$ and then $A \rightarrow \infty$ in (3.20), in view of the bounds (3.1), (3.3), and (3.17) and the fact that $u=0$ on $\Gamma$, we obtain that

$$
u(x)=\int_{\Gamma} \partial_{n} u(y) G(x, y) d s(y)+R_{a},
$$

where the integral over $\Gamma$ is well defined by (3.13) and (3.16), and

$$
R_{a}=\int_{\Gamma_{a}}\left[u(y) \frac{\partial G(x, y)}{\partial n(y)}-G(x, y) \frac{\partial u}{\partial n}(y)\right] d s(y) .
$$

Since $u$ satisfies the UPRC and $G(x, \cdot)$ is a radiating solution in $U_{h}$ for $h>x_{2}$, we find, in view of (3.16) and the equivalence of (i) and (v) in Lemma 2.1, that $R_{a}=0$.

3.4. The uniqueness result. Let $u$ be a solution of Problem (P) (with $g \equiv 0$, $k>0$ ), and let $a>f_{+}$. Then, by Theorem 3.2 with $B=-A$,

$$
K_{A} \equiv \int_{\Gamma(A)}\left|\partial_{n} u\right|^{2} d s \leq C\left\{I_{A}+R_{1}(A)\right\}, \quad A>0,
$$

where $I_{A}=I(-A, A)$ and $R_{1}(A)=R_{1}(-A, A)$ with $I(-A, A)$ and $R_{1}(-A, A)$ as defined in section 3.2 .

To use the inequality (3.22) we need the following two lemmas. The first of these is a consequence of the UPRC and was proved in [11, Lemma 6.1].

Lemma 3.2. If $\phi \in L_{2}\left(\Gamma_{h}\right) \cap L_{\infty}\left(\Gamma_{h}\right)$ and $v$ is defined by (2.3), then the restrictions of $v, \partial_{1} v$, and $\partial_{2} v$ to $\Gamma_{a}$ are in $L_{2}\left(\Gamma_{a}\right) \cap B C\left(\Gamma_{a}\right)$ for all $a>h$ and

$$
\int_{\Gamma_{a}}\left[\left|\partial_{2} v\right|^{2}-\left|\partial_{1} v\right|^{2}+k^{2}|v|^{2}\right] d s \leq 2 k \Im \int_{\Gamma_{a}} \bar{v} \partial_{2} v d s .
$$

Lemma 3.3. Suppose that u satisfies the conditions of Theorem 3.2. Then

$$
J_{A}=R_{2}(A), \quad A>0
$$

where

$$
J_{A}=\Im \int_{\Gamma_{a}(A)} \bar{u} \partial_{2} u d s, \quad R_{2}(A)=\Im\left[\int_{\gamma_{a}(-A)}-\int_{\gamma_{a}(A)}\right] \bar{u} \partial_{1} u d s .
$$

Proof. Apply Green's first theorem to $u$ and $\bar{u}$ in $S=\left\{x \in D_{a}(A) \mid x_{2}>f\left(x_{1}\right)+\epsilon\right\}$ with $\epsilon>0$ to obtain that

$$
\int_{S}\left\{|\nabla u|^{2}-k^{2}|u|^{2}\right\} d x=\int_{\partial S} \bar{u} \partial_{n} u d s
$$


on noting that $\Delta u=-k^{2} u$ in $D$. Taking the imaginary part of this equation and then the limit $\epsilon \rightarrow 0$ we obtain (3.24) on utilizing the bounds in Theorem 3.1.

To make use of Lemma 3.2 we define

$$
v(x)=\int_{\Gamma(A)} \partial_{n} u(y) G(x, y) d s(y), \quad x \in D .
$$

Then, by (3.18) and Corollary 3.1 combined with the Cauchy-Schwarz inequality, we have that $\left.v\right|_{\Gamma_{b}}$, the restriction of $v$ to $\Gamma_{b}$, is in $L_{2}\left(\Gamma_{b}\right) \cap B C\left(\Gamma_{b}\right)$ for all $b>f_{+}$. On the other hand, $v$ is a radiating solution in $U_{b}$ for $b>f_{+}$, so that, in view of the equivalence of (ii) and (iv) in Lemma 2.1, $v$ satisfies (2.3) with $h=b$ and $\phi=\left.v\right|_{\Gamma_{b}}$ for every $b>f_{+}$. Set

$$
\begin{gathered}
J_{A}^{\prime}=\Im \int_{\Gamma_{a}(A)} \bar{v} \partial_{2} v d s, \quad J_{A}^{\prime \prime}=\Im \int_{\Gamma_{a}} \bar{v} \partial_{2} v d s \\
I_{A}^{\prime}=\int_{\Gamma_{a}(A)}\left\{\left|\partial_{2} v\right|^{2}-\left|\partial_{1} v\right|^{2}+k^{2}|v|^{2}\right\} d s, \quad I_{A}^{\prime \prime}=\int_{\Gamma_{a}}\left\{\left|\partial_{2} v\right|^{2}-\left|\partial_{1} v\right|^{2}+k^{2}|v|^{2}\right\} d s .
\end{gathered}
$$

Then, by Lemma 3.2 ,

$$
I_{A}^{\prime \prime} \leq 2 k J_{A}^{\prime \prime},
$$

so that, by (3.22) and Lemma 3.3,

$$
K_{A} \leq C\left[\left(I_{A}-I_{A}^{\prime \prime}\right)+2 k\left(J_{A}^{\prime \prime}-J_{A}\right)+R_{1}(A)+2 k R_{2}(A)\right] .
$$

Now set $w\left(x_{1}\right)=\partial_{n} u\left(\left(x_{1}, f\left(x_{1}\right)\right)\right), x_{1} \in \mathbf{R}$. Then, for all $A>0$,

$$
\int_{-A}^{A}\left|w\left(x_{1}\right)\right|^{2} d x_{1} \leq K_{A} \leq\left(1+L^{2}\right)^{1 / 2} \int_{-A}^{A}\left|w\left(x_{1}\right)\right|^{2} d x_{1} .
$$

By (3.18), (3.19), and (3.25),

$$
\begin{aligned}
|v(x)|,|\nabla v(x)| & \leq C_{a}\left(1+L^{2}\right)^{1 / 2} W_{A}\left(x_{1}\right), \quad x \in \Gamma_{a}, \\
|u(x)-v(x)|,|\nabla u(x)-\nabla v(x)| & \leq C_{a}\left(1+L^{2}\right)^{1 / 2}\left(W_{\infty}\left(x_{1}\right)-W_{A}\left(x_{1}\right)\right), \quad x \in \Gamma_{a},
\end{aligned}
$$

where, for $0 \leq A \leq+\infty$,

$$
W_{A}\left(x_{1}\right)=\int_{-A}^{A}\left(1+\left|x_{1}-y_{1}\right|\right)^{-3 / 2}\left|w\left(y_{1}\right)\right| d y_{1}, \quad x_{1} \in \mathbf{R} .
$$

It follows that

$$
\left|I_{A}^{\prime}-I_{A}^{\prime \prime}\right|,\left|J_{A}^{\prime}-J_{A}^{\prime \prime}\right| \leq C \int_{\mathbf{R} \backslash[-A, A]}\left(W_{A}\left(x_{1}\right)\right)^{2} d x_{1},
$$

where $C=C_{a}^{2}\left(1+L^{2}\right)\left(2+k^{2}\right)$, and that

$$
\left|I_{A}-I_{A}^{\prime}\right|,\left|J_{A}-J_{A}^{\prime}\right| \leq 2 C \int_{-A}^{A}\left(W_{\infty}\left(x_{1}\right)-W_{A}\left(x_{1}\right)\right) W_{\infty}\left(x_{1}\right) d x_{1},
$$

so that, for some constant $c>0$ and all $A>0$,

$$
\begin{aligned}
\int_{-A}^{A}\left|w\left(x_{1}\right)\right|^{2} d x_{1} \leq c\{ & \int_{\mathbf{R} \backslash[-A, A]} W_{A}^{2}\left(x_{1}\right) d x_{1}+ \\
& \left.\int_{-A}^{A}\left(W_{\infty}\left(x_{1}\right)-W_{A}\left(x_{1}\right)\right) W_{\infty}\left(x_{1}\right) d x_{1}+\left|R_{1}(A)\right|+\left|R_{2}(A)\right|\right\} .
\end{aligned}
$$


Further, by Corollary 3.1,

$$
\sup _{j \in \mathbf{Z}} \int_{j-1}^{j}\left|w\left(x_{1}\right)\right|^{2} d x_{1}<+\infty
$$

so that, applying Lemma $\mathrm{A}$ in the appendix, we obtain that $w \in L_{2}(\mathbf{R})$ (equivalently, by $\left.(3.26), \partial_{n} u \in L_{2}(\Gamma)\right)$ and, for all $A_{0}>0$,

$$
\left(1+L^{2}\right)^{-1 / 2} \int_{\Gamma}\left|\partial_{n} u\right|^{2} d s \leq \int_{-\infty}^{\infty}\left|w\left(x_{1}\right)\right|^{2} d x_{1} \leq c \sup _{A>A_{0}}\left(\left|R_{1}(A)\right|+\left|R_{2}(A)\right|\right) .
$$

For $x \in D_{a}$ with $\left|x_{1}\right| \geq 1$, we have, by (3.19) and (3.16) along with the CauchySchwarz inequality,

$$
\begin{aligned}
|u(x)|^{2} \leq & 2\left\{\int_{\Gamma \backslash \Gamma\left(\left|x_{1}\right| / 2\right)}\left|\partial_{n} u(y)\right||G(x, y)| d s(y)\right\}^{2} \\
& +2\left\{\int_{\Gamma\left(\left|x_{1}\right| / 2\right)}\left|\partial_{n} u(y)\right||G(x, y)| d s(y)\right\}^{2} \\
\leq & C_{1} \int_{\Gamma \backslash \Gamma\left(\left|x_{1}\right| / 2\right)}\left|\partial_{n} u\right|^{2} d s+C_{2}\left\{\frac{\left|x_{1}\right|}{2}\right\}^{-3},
\end{aligned}
$$

where

$$
C_{1}=2 \sup _{x \in D_{a}} \int_{\Gamma}|G(x, y)|^{2} d s(y)<\infty
$$

by (3.15) and (3.16) and

$$
C_{2}=8 C^{2} a^{4} \int_{\Gamma}\left|\partial_{n} u\right|^{2} d s
$$

with $C$ as given in (3.16). Thus, $u(x) \rightarrow 0$ as $x_{1} \rightarrow \infty$ with $x \in D_{a}$, uniformly in $x_{2}$. Noting also Theorem 3.1 and Lemma 3.1, it follows that $R_{j}(A) \rightarrow 0$ as $A \rightarrow \infty$, $j=1,2$ and thus, from (3.28), that $\partial_{n} u=0$ on $\Gamma$ and hence, from (3.19), that $u \equiv 0$ in $D$. We have shown the following result on noting the remark made at the beginning of section 3 .

Theorem 3.4. Problem (P) has at most one solution.

4. Existence of solution. We require additionally in this section that $f^{\prime}$ is uniformly Hölder continuous, i.e., that, for some constants $M, \alpha>0$,

$$
\left|f^{\prime}(s)-f^{\prime}(t)\right| \leq M|s-t|^{\alpha}, \quad s, t \in \mathbf{R},
$$

so that the whole boundary $\Gamma$ is Lyapunov. With this additional assumption we apply the results of [10] to show existence of solution to Problem $(\mathrm{P})$ when $\Gamma$ is a small perturbation of a flat boundary.

Let

$$
G_{1}(x, y)=\Phi(x, y)+\Phi\left(x, y^{\prime}\right)+\hat{P}\left(x-y^{\prime}\right), \quad x, y \in \overline{U_{0}}, \quad x \neq y,
$$

where $y=\left(y_{1}, y_{2}\right), y^{\prime}=\left(y_{1},-y_{2}\right)$, and

$$
\hat{P}(x):=\frac{e^{i k|x|}}{\pi} \int_{0}^{\infty} \frac{t^{-1 / 2} e^{-k|x| t}(1+\gamma(1+i t))}{\sqrt{t-2 i}(t-i(1+\gamma))^{2}} d t, \quad x \in \overline{U_{0}}
$$


with $\gamma=x_{2} /|x|$. Then (see $\left.[8]\right), \hat{P} \in C\left(\overline{U_{0}}\right) \cap C^{\infty}\left(\overline{U_{0}} \backslash\{0\}\right)$ and $G_{1}(x, y)$ is the Green's function for the operator $\Delta+k^{2}$, which satisfies the impedance boundary condition

$$
\frac{\partial G_{1}(x, y)}{\partial x_{2}}+i k G_{1}(x, y)=0, \quad x \in \Gamma_{0}, y \in \overline{U_{0}}, x \neq y .
$$

Chandler-Wilde and Ross [10] suggested looking for a solution to Problem (P)(i)-(iii) in the form of a double layer potential,

$$
u(x)=\int_{\Gamma} \frac{\partial G_{1}(x, y)}{\partial n(y)} \psi(y) d s(y), \quad x \in D,
$$

for some $\psi \in B C(\Gamma)$. It is shown in [10] that, for every $C>0$,

$$
G_{1}(x, y), \nabla_{y} G_{1}(x, y)=O\left(|x-y|^{-3 / 2}\right)
$$

uniformly in $x$ and $y$ with $0 \leq x_{2}, y_{2} \leq C$ as $|x-y| \rightarrow \infty$, so that (4.4) is well defined. It is easy to see using (4.3) [5] that $\hat{P}$ is a radiating solution in $U_{0}$, and hence

$$
u_{m}(x)=\int_{\Gamma(m)} \frac{\partial G_{1}(x, y)}{\partial n(y)} \psi(y) d s(y), \quad x \in D,
$$

is a radiating solution in $U_{h}$ for every $h>f_{+}$and $m \in \mathbf{N}$. Using (4.5) we see that $u_{m}(x) \rightarrow u(x)$ as $m \rightarrow \infty$ uniformly on compact subsets of $U_{h}$, and, by [10, Lemma $4.2]$,

$$
\sup _{m \in \mathbf{N}, x \in U_{h}} x_{2}^{-1 / 2}\left|u_{m}(x)\right|<\infty .
$$

Thus, by the equivalence of (i) and (ii) in Lemma 2.1, $u$, given by (4.4), satisfies the UPRC. Combining this result with Lemmas 4.1-4.3 in [10] we have the following result.

Theorem 4.1. The double-layer potential (4.4) satisfies Problem $(\mathrm{P})$, with $\beta=$ $-1 / 2$ in (2.9), provided $\psi \in B C(\Gamma)$ satisfies the boundary integral equation

$$
\psi(x)=2 \int_{\Gamma} \frac{\partial G_{1}(x, y)}{\partial n(y)} \psi(y) d s(y)-2 g(x), \quad x \in \Gamma .
$$

It is shown in [10, Theorem 5.5] that the integral equation (4.7) has exactly one solution provided $\Gamma$ is sufficiently close to a straight line. Combining Theorem 4.1 with [10, Theorem 5.5] we obtain Theorem 4.2 below. In this theorem, to make explicit the dependence on the wavenumber $k, u^{(\lambda)}$ denotes the unique solution (if it exists) of Problem (P) when $k=\lambda$.

TheOREM 4.2. For every $H>0$ there exists a constant $\epsilon>0$ (dependent on $H$ and $M$ ) such that, if

$$
|f(s)-H| \leq \epsilon,\left|f^{\prime}(s)\right| \leq \epsilon, \quad s \in \mathbf{R},
$$

then (4.7) has exactly one solution $\psi \in B C(\Gamma)$ for every $g \in B C(\Gamma)$. Further, $u$, given by (4.4), is the unique solution of Problem (P) and, for some constant $C>0$ independent of $g$,

$$
|u(x)| \leq C x_{2}^{1 / 2}\|g\|_{\infty}, \quad x \in D
$$


Moreover, in the case $k>0$, u satisfies the following limiting absorption principle: that, for all $\epsilon>0$ sufficiently small, $u^{(k+i \epsilon)}$ exists and $u^{(k+i \epsilon)}(x) \rightarrow u(x)$ as $\epsilon \rightarrow 0$, uniformly on compact subsets of $\bar{D}$.

We remark that an example in [7] for the case of a flat boundary shows that, for particular choices of $g$, the solution of Problem (P) does not satisfy (2.9) for $\beta>-1 / 2$, so that the exponent $1 / 2$ in (4.8) gives a sharp bound for the general case.

Appendix. The following lemma is a key element in the uniqueness proof of the paper. It has also been used recently as part of the uniqueness proof for a related problem of scattering by an infinite inhomogeneous layer [11], and it is anticipated that it will prove valuable as an ingredient in the proof of many other uniqueness results for scattering by one-dimensional rough surfaces and interfaces. The proof of the lemma is suggested in large part by the proofs of Lemmas 4.8-4.14 in [7].

Lemma A. Suppose that $F \in L_{2}^{\text {loc }}(\mathbf{R})$ and that, for some nonnegative constants $M, C, \epsilon$, and $A_{0}$,

$$
\int_{j-1}^{j}|F(t)|^{2} d t \leq M^{2}, \quad j \in \mathbf{Z}
$$

and

$$
\begin{aligned}
& \int_{-A}^{A}|F(t)|^{2} d t \\
\leq & C \int_{\mathbf{R} \backslash[-A, A]} G_{A}^{2}(t) d t+C \int_{-A}^{A}\left[G_{\infty}(t)-G_{A}(t)\right] G_{\infty}(t) d t+\epsilon, \quad A>A_{0},
\end{aligned}
$$

where, for $0<A \leq+\infty$,

$$
G_{A}(s)=\int_{-A}^{A}(1+|s-t|)^{-3 / 2}|F(t)| d t, \quad s \in \mathbf{R} .
$$

Then $F \in L_{2}(\mathbf{R})$ and

$$
\int_{-\infty}^{+\infty}|F(t)|^{2} d t \leq \epsilon
$$

Proof. For $A>A_{0}$ let

$$
J_{A}=\int_{-A}^{A}|F(t)|^{2} d t, \quad P_{A}=\int_{\mathbf{R} \backslash I_{1}} G_{A}^{2}(t) d t, \quad Q_{A}=\int_{-A}^{A}\left[G_{\infty}(t)-G_{A}(t)\right] G_{\infty}(t) d t,
$$

where $I_{\alpha}=\left[-A^{\alpha}, A^{\alpha}\right]$ for $\alpha>0$. We will show, in a number of steps, that $P_{A} \rightarrow 0$, $Q_{A} \rightarrow 0$ as $A \rightarrow \infty$ through some sequence of positive values. It is clear that it then follows from (A.2) that $F \in L_{2}(\mathbf{R})$. Further, by taking the limit $A \rightarrow \infty$ through this sequence in (A.2), we obtain (A.4). Throughout the proof $C$ denotes a positive constant independent of $A$, not necessarily the same at each occurence.

Step 1. We first show that $P_{A}=O(\ln A)$ as $A \rightarrow \infty$.

Noting that $|s-j-1 / 2|-1 / 2 \leq|s-t| \leq|s-j-1 / 2|+1 / 2$ for $s \in \mathbf{R}, j-1 \leq t \leq j$, we see, using the Cauchy-Schwarz inequality, that, for $j \in \mathbf{Z}$,

$$
\int_{j-1}^{j}(1+|s-t|)^{-3 / 2}|F(t)| d t \leq(1 / 2+|s-j-1 / 2|)^{-3 / 2} M
$$




$$
\begin{aligned}
& \leq 2^{3 / 2} M(1+|s-j-1 / 2|)^{-3 / 2} \\
& \leq 8 M \int_{j-1}^{j}(1+|s-t|)^{-3 / 2} d t .
\end{aligned}
$$

Thus, for $s \in \mathbf{R}$,

$$
G_{A}(s) \leq 8 M \int_{-A-1}^{A+1}(1+|s-t|)^{-3 / 2} d t, \quad A>A_{0},
$$

and

$$
G_{A}(s) \leq 16 M \int_{0}^{\infty}(1+t)^{-3 / 2} d t, \quad A_{0} \leq A \leq+\infty
$$

so that, for $|s| \geq A+1, A>A_{0}$,

$$
\begin{aligned}
G_{A}(s) & \leq C \int_{-A-1}^{A+1}(1+|s|-t)^{-3 / 2} d t \leq C\left\{(|s|-A)^{-1 / 2}-(2+|s|+A)^{-1 / 2}\right\} \\
& \leq C(A+1)(|s|-A)^{-1 / 2}(2+|s|+A)^{-1}
\end{aligned}
$$

since, for all $b \geq a>0$, we have $a^{-1 / 2}-b^{-1 / 2} \leq a^{-1 / 2} b^{-1}(b-a)$. Thus, and using (A.7) to bound $G_{A}(s)$ for $A \leq|s|<A+1$, we obtain that, for $A>A_{0}$,

$$
\begin{aligned}
P_{A} & \leq C\left\{1+(A+1)^{2} \int_{A+1}^{\infty}(s-A)^{-1}(2+s+A)^{-2} d s\right\} \\
& =C\left\{1+\int_{1 /(A+1)}^{\infty} \frac{d t}{t(2+t)^{2}}\right\},
\end{aligned}
$$

substituting $1+s=(A+1)(1+t)$. Thus $P_{A}=O(\ln A)$ as $A \rightarrow \infty$.

Step 2 . We next show by induction that $J_{A}=O\left(A^{2 / n}\right)$ as $A \rightarrow \infty$ for $n=2,3, \ldots$.

By (A.1) this is true for $n=2$. Suppose now that $J_{A}=O\left(A^{2 / k}\right)$ as $A \rightarrow \infty$ for some integer $k \geq 2$. Set $p=2 / k, \alpha=2 /(p+1)$. We have that

$$
G_{\infty}(s)-G_{A}(s)=\int_{\mathbf{R} \backslash I_{1}}(1+|s-t|)^{-3 / 2}|F(t)| d t, \quad s \in \mathbf{R} .
$$

Further, for $|s| \leq A$, by the Cauchy-Schwarz inequality,

$$
\begin{aligned}
\int_{I_{\alpha} \backslash I_{1}}(1+|s-t|)^{-3 / 2}|F(t)| d t & \leq J_{A^{\alpha}}^{1 / 2}\left\{\int_{I_{\alpha} \backslash I_{1}} \frac{d t}{(1+|s-t|)^{3}}\right\}^{1 / 2} \\
& \leq C A^{\alpha p / 2}\left\{\int_{A}^{\infty} \frac{d t}{(1+|s-t|)^{3}}\right\}^{1 / 2} \leq \frac{C A^{p /(p+1)}}{1+A-|s|} .
\end{aligned}
$$

Also, using (A.5), for $|s| \leq A$ and all $A$ sufficiently large,

$$
\begin{aligned}
\int_{\mathbf{R} \backslash I_{\alpha}}(1+|s-t|)^{-3 / 2}|F(t)| d t & \leq 8 M \int_{\mathbf{R} \backslash\left[1-A^{\alpha}, A^{\alpha}-1\right]}(1+|s-t|)^{-3 / 2} d t \\
& \leq C \int_{A^{\alpha}-1}^{\infty}(1+t-|s|)^{-3 / 2} d t \leq C\left(A^{\alpha}-|s|\right)^{-1 / 2}
\end{aligned}
$$


Now

$$
\int_{-A}^{A} \frac{d s}{1+A-|s|}=2 \ln (A+1)
$$

and

$$
\int_{-A}^{A} \frac{d s}{\left(A^{\alpha}-|s|\right)^{1 / 2}}=4\left\{A^{\alpha / 2}-\left(A^{\alpha}-A\right)^{1 / 2}\right\} \leq 4 A^{1-\alpha / 2}=4 A^{p /(p+1)} .
$$

Thus, and using (A.7), $Q_{A}=O\left(A^{p /(p+1)} \ln A\right)$ as $A \rightarrow \infty$ and, since $p /(p+1)<$ $2 p /(p+2)=2 /(k+1), Q_{A}=O\left(A^{2 /(k+1)}\right)$ as $A \rightarrow \infty$ and $J_{A}=O\left(A^{2 /(k+1)}\right)$ as $A \rightarrow \infty$ by (A.2) and Step 1 .

Step 3. We show that $F^{(\epsilon)}, G_{\infty}^{(\epsilon)} \in L_{2}(\mathbf{R})$ for all $\epsilon>0$, where $F^{(\epsilon)}(s)=(1+$ $|s|)^{-\epsilon} F(s), G_{\infty}^{(\epsilon)}(s)=(1+|s|)^{-\epsilon} G_{\infty}(s)$.

Clearly, it is sufficient to show that this holds for $\epsilon$ in the range $0<\epsilon<1 / 2$. We have that $J_{A}=O\left(A^{\epsilon}\right)$ as $A \rightarrow \infty$ by Step 2 . Integrating by parts,

$$
\begin{aligned}
\int_{-A}^{A}\left(1+t^{2}\right)^{-\epsilon}|F(t)|^{2} d t & =\left(1+A^{2}\right)^{-\epsilon} \int_{-A}^{A}|F(t)|^{2} d t \\
& +2 \epsilon \int_{-A}^{A}\left\{\int_{0}^{t}|F(s)|^{2} d s\right\} t\left(1+t^{2}\right)^{-1-\epsilon} d t \\
\leq & J_{A}\left(1+A^{2}\right)^{-\epsilon}+2 \epsilon \int_{-A}^{A} t\left(1+t^{2}\right)^{-1-\epsilon} J_{t} d t=O(1)
\end{aligned}
$$

as $A \rightarrow \infty$ since $t\left(1+t^{2}\right)^{-1-\epsilon} J_{t}=O\left(t^{-1-\epsilon}\right)$ as $t \rightarrow \infty$. It follows that $F^{(\epsilon)} \in L_{2}(\mathbf{R})$. Since $(1+|t|) /(1+|s|) \leq 1+|t-s|, t, s \in \mathbf{R}$, we have that

$$
G_{\infty}^{(\epsilon)}(s) \leq \int_{-\infty}^{\infty}(1+|s-t|)^{\epsilon-3 / 2}\left|F^{(\epsilon)}(t)\right| d t
$$

and thus $G_{\infty}^{(\epsilon)} \in L_{2}(\mathbf{R})$ by Young's theorem.

Step 4 . We show that $P_{A} \rightarrow 0$ as $A \rightarrow \infty$ through some sequence of positive values.

Since, by Step 2, $J_{A}=O\left(A^{\epsilon}\right)$ as $A \rightarrow \infty$ for all $\epsilon>0$, it follows from [4, Lemma $4.12]$ that, given $\alpha$ in the range $0<\alpha<1 / 2$, there exists a sequence $\left\{A_{m} \mid m \in \mathbf{N}\right\} \subset$ $\left[A_{0}+1, \infty\right)$ such that $A_{m} \rightarrow \infty$ as $m \rightarrow \infty$ and

$$
\int_{W_{m}}|F(t)|^{2} d t \leq C A_{m}^{-\alpha}, \quad m \in \mathbf{N},
$$

where, for $m \in \mathbf{N}, W_{m}=W_{m}^{+} \cup W_{m}^{-}$,

$$
W_{m}^{+}=\left[-A_{m}^{+}, A_{m}^{+}\right] \backslash\left[-A_{m}, A_{m}\right], \quad W_{m}^{-}=\left[-A_{m}, A_{m}\right] \backslash\left[-A_{m}^{-}, A_{m}^{-}\right],
$$

and $A_{m}^{ \pm}=A_{m} \pm A_{m}^{1 / 2}$.

Now, by the Cauchy-Schwarz inequality, for $|s|>A_{m}$,

$$
\begin{aligned}
\int_{-A_{m}^{-}}^{A_{m}^{-}} \frac{|F(t)| d t}{(1+|s-t|)^{3 / 2}} & \leq J_{A_{m}^{-}}^{1 / 2}\left\{\int_{-A_{m}^{-}}^{A_{m}^{-}} \frac{d t}{(1+|s|-|t|)^{3}}\right\}^{1 / 2} \\
& \leq C J_{A_{m}}^{1 / 2}\left(1+|s|-A_{m}^{-}\right)^{-1} .
\end{aligned}
$$


Similarly, and using (A.9), for $|s|>A_{m}$,

$$
\begin{aligned}
\int_{W_{m}^{-}}(1+|s-t|)^{-3 / 2}|F(t)| d t & \leq\left\{\int_{W_{m}^{-}}|F(t)|^{2} d t\right\}^{1 / 2}\left\{\int_{W_{m}^{-}}(1+|s|-|t|)^{-3} d t\right\}^{1 / 2} \\
& \leq C A_{m}^{-\alpha / 2}\left(1+|s|-A_{m}\right)^{-1} .
\end{aligned}
$$

Thus, and since $J_{A}=O\left(A^{\epsilon}\right)$ as $A \rightarrow \infty$ for all $\epsilon>0$, for $|s|>A_{m}$ we have

$$
\begin{aligned}
G_{A_{m}}^{2}(s) & \leq C\left\{A_{m}^{\epsilon / 2}\left(1+|s|-A_{m}^{-}\right)^{-1}+A_{m}^{-\alpha / 2}\left(1+|s|-A_{m}\right)^{-1}\right\}^{2} \\
& \leq C A_{m}^{\epsilon}\left(1+|s|-A_{m}^{-}\right)^{-2}+C A_{m}^{-\alpha}\left(1+|s|-A_{m}\right)^{-2} .
\end{aligned}
$$

Thus

$$
\begin{aligned}
P_{A_{m}} & \leq C A_{m}^{\epsilon} \int_{A_{m}}^{\infty} \frac{d s}{\left(1+s-A_{m}^{-}\right)^{2}}+C A_{m}^{-\alpha} \int_{A_{m}}^{\infty} \frac{d s}{\left(1+s-A_{m}\right)^{2}} \\
& =C A_{m}^{\epsilon}\left(1+A_{m}^{1 / 2}\right)^{-1}+C A_{m}^{-\alpha} \rightarrow 0
\end{aligned}
$$

as $m \rightarrow \infty$ on choosing $\epsilon<1 / 2$.

Step 5 . We show that $Q_{A_{m}} \rightarrow 0$ as $m \rightarrow \infty$, where $\left\{A_{m}\right\}$ is the sequence in Step 4 .

By the Cauchy-Schwarz inequality, for $|s| \leq A_{m}$ and $0<\epsilon<1 / 2$,

$$
\begin{aligned}
\int_{\mathbf{R} \backslash\left[-A_{m}^{+}, A_{m}^{+}\right]} \frac{|F(t)|}{(1+|s-t|)^{3 / 2}} d t & \leq \sqrt{2}\left\|F^{(\epsilon)}\right\|_{2}\left\{\int_{A_{m}^{+}}^{\infty} \frac{(1+t)^{2 \epsilon} d t}{(1+t-|s|)^{3}}\right\}^{1 / 2} \\
& \leq C A_{m}^{\epsilon / 2}\left\{\int_{A_{m}^{+}}^{\infty}(1+t-|s|)^{2 \epsilon-3} d t\right\}^{1 / 2} \\
& \leq C A_{m}^{\epsilon / 2}\left(1+A_{m}^{+}-|s|\right)^{\epsilon-1} .
\end{aligned}
$$

Similarly, and using (A.9), for $|s| \leq A_{m}$,

$$
\begin{aligned}
\int_{W_{m}^{+}}(1+|s-t|)^{-3 / 2}|F(t)| d t & \leq\left\{\int_{W_{m}^{+}}|F(t)|^{2} d t\right\}^{1 / 2}\left\{\int_{W_{m}^{+}}(1+|t|-|s|)^{-3} d t\right\}^{1 / 2} \\
& \leq C A_{m}^{-\alpha / 2}\left(1+A_{m}-|s|\right)^{-1} .
\end{aligned}
$$

Thus, and noting (A.8),

$$
\begin{aligned}
{\left[G_{\infty}(s)-G_{A_{m}}(s)\right]^{2} } & \leq C\left[A_{m}^{\epsilon / 2}\left(1+A_{m}^{+}-|s|\right)^{\epsilon-1}+A_{m}^{-\alpha / 2}\left(1+A_{m}-|s|\right)^{-1}\right]^{2} \\
& \leq C A_{m}^{\epsilon}\left(1+A_{m}^{+}-|s|\right)^{2 \epsilon-2}+C A_{m}^{-\alpha}\left(1+A_{m}-|s|\right)^{-2} .
\end{aligned}
$$

Now

$$
\int_{-A_{m}}^{A_{m}} G_{\infty}^{2}(t) d t \leq\left(1+A_{m}\right)^{2 \epsilon} \int_{-A_{m}}^{A_{m}}(1+|t|)^{-2 \epsilon} G_{\infty}^{2}(t) d t \leq\left(1+A_{m}\right)^{2 \epsilon}\left\|G_{\infty}^{(\epsilon)}\right\|_{2}^{2},
$$

and so, by the Cauchy-Schwarz inequality,

$$
\begin{aligned}
Q_{A_{m}} & \leq C A_{m}^{\epsilon}\left\{A_{m}^{\epsilon} \int_{-A_{m}}^{A_{m}}\left(1+A_{m}^{+}-|s|\right)^{2 \epsilon-2} d s+A_{m}^{-\alpha} \int_{-A_{m}}^{A_{m}}\left(1+A_{m}-|s|\right)^{-2} d s\right\}^{1 / 2} \\
& \leq C A_{m}^{\epsilon}\left\{A_{m}^{\epsilon}\left(1+A_{m}^{+}-A_{m}\right)^{2 \epsilon-1}+A_{m}^{-\alpha}\right\}^{1 / 2} \leq C A_{m}^{-1 / 8}
\end{aligned}
$$

on choosing $\epsilon=1 / 16$ and $\alpha=3 / 8$.

In view of the preliminary remarks made before Step 1 , the proof is complete. 


\section{REFERENCES}

[1] G. BaO, D. C. Dobson, And J. A. Cox, Mathematical studies in rigorous grating theory, J. Opt. Soc. Amer. A, 12 (1995), pp. 1029-1042.

[2] A. Benali, J. Chandezon, and J. Fontaine, A new theory for scattering of electromagnetic waves from conducting or dielectric rough surfaces, IEEE Trans. Antennas and Propagation, 40 (1992), pp. 141-148.

[3] M. Cadilhac, Some mathematical aspects of the grating theory, in Electromagnetic Theory of Gratings, R. Petit, ed., Springer-Verlag, Berlin, 1980, pp. 509-535.

[4] P. CAO AND C. MaCASKILL, Iterative techniques for rough surface scattering problems, Wave Motion, 21 (1995), pp. 209-229.

[5] S. N. Chandler-Wilde, Ground Effects in Environmental Sound Propagation, Ph.D. thesis, Department of Civil Engineering, University of Bradford, UK, 1988.

[6] S. N. Chandler-Wilde, Boundary value problems for the Helmholtz equation in a half-plane, in Proc. 3rd Int. Conf. on Mathematical and Numerical Aspects of Wave Propagation, G. Cohen, ed., SIAM, Philadelphia, 1995, pp. 188-197.

[7] S. N. ChANDLER-WILDE, The impedance boundary value problem for the Helmholtz equation in a half-plane, Math. Methods Appl. Sci., 20 (1997), pp. 813-840.

[8] S. N. Chandler-Wilde and D. C. Hothersall, Efficient calculation of the Green function for acoustic propagation above a homogeneous impedance plane, J. Sound Vibration, 180 (1995), pp. 705-724.

[9] S. N. Chandler-Wilde And C.R. Ross, Uniqueness results for direct and inverse scattering by infinite surfaces in a lossy medium, Inverse Problems, 10 (1995), pp. 1063-1067.

[10] S. N. Chandler-Wilde And C.R. Ross, Scattering by rough surfaces: the Dirichlet problem for the Helmholtz equation in a non-locally perturbed half-plane, Math. Methods Appl. Sci., 19 (1996), pp. 959-976.

[11] S. N. ChandleR-Wilde And Bo Zhang, Electromagnetic scattering by an inhomogeneous conducting or dielectric layer on a perfectly conducting plate, Proc. Roy. Soc. London Ser. A, 454 (1998), pp. 519-542.

[12] J. A. DeSAnto, Exact spectral formalism for rough-surface scattering, J. Opt. Soc. Amer. A, 2 (1985), pp. 2202-2207.

[13] J. A. DeSanto and G. S. Brown, Analytical techniques for multiple scattering from rough surfaces, in Progress in Optics XXIII, E. Wolf, ed., Elsevier, New York, 1986, pp. 1-62.

[14] D. Gilbarg and N.S. Trudinger, Elliptic Partial Differential Equations of Second Order, 2nd ed., Springer-Verlag, Berlin, 1983.

[15] P. GRISVARD, Behavior of the solutions of an elliptic boundary value problem in a polygonal or polyhedral domain, in Numerical Solution of Partial Differential Equations III, B. Hubbard, ed., Academic Press, New York, 1976, pp. 207-274.

[16] M. J. Kim, H. M. Berenyi, ANd R. E. BuRge, Scattering of scalar waves by two-dimensional gratings of arbitrary shape: application to rough surfaces at near-grazing incidence, Proc. Roy. Soc. Lond. Ser. A, 446 (1994), pp. 289-308.

[17] A. Kirsch, Diffraction by periodic structures, in Inverse Problems in Mathematical Physics, L. Paivarinta and E. Somersalo, eds., Springer-Verlag, Berlin, 1993, pp. 87-102.

[18] R. Petit, Electromagnetic Theory of Gratings, Springer-Verlag, Berlin, 1980.

[19] M. SPIVACK, A numerical approach to rough-surface scattering by the parabolic equation method, J. Acoust. Soc. Am., 87 (1990), pp. 1999-2004.

[20] K. O. Widman, Inequalities for the Green function and boundary continuity of the gradient of solutions of elliptic differential equations, Math. Scand., 21 (1967), pp. 17-37. 